\title{
Identification of miR-194-5p as a potential biomarker for postmenopausal osteoporosis
}

Jia Meng, Dapeng Zhang, Nanan Pan, Ning Sun, Qiujun Wang, Jingxue Fan, Ping Zhou, Wenliang Zhu, Lihong Jiang

The incidence of osteoporosis is high in postmenopausal women due to altered estrogen levels and continuous calcium loss that occurs with aging. Recent studies have shown that microRNAs (miRNAs) are involved in the development of osteoporosis. These miRNAs may be used as potential biomarkers to identify women at a high risk for developing the disease. In this study, whole blood samples were collected from 48 postmenopausal Chinese women with osteopenia or osteoporosis and pooled into six groups according to individual T-scores. A miRNA microarray analysis was performed on pooled blood samples to identify potential miRNA biomarkers for postmenopausal osteoporosis. Five miRNAs (miR-130b-3p, -151a-3p, -151b, -194-5p, and -590-5p) were identified in the microarray analysis. These dysregulated miRNAs were subjected to a pathway analysis investigating whether they were involved in regulating osteoporosis-related pathways. Among them, only miR-194-5p was enriched in multiple osteoporosis-related pathways. Enhanced miR194-5p expression in women with osteoporosis was confirmed by quantitative reverse transcription-polymerase chain reaction analysis. For external validation, a significant correlation between the expression of miR-194-5p and T-scores was found in an independent patient collection comprised of 24 postmenopausal women with normal bone mineral density, 30 postmenopausal women with osteopenia, and 32 postmenopausal women with osteoporosis $(p<0.05)$. Taken together, the present findings suggest that miR-194-5p may be a viable miRNA biomarker for postmenopausal osteoporosis. 
3 Jia Meng ${ }^{1 *}$, Dapeng Zhang ${ }^{2 *}$, Nanan Pan ${ }^{1}$, Ning Sun ${ }^{3}$, Qiujun Wang ${ }^{1}$, Jingxue Fan ${ }^{1}$, Ping Zhou ${ }^{1}$, Wenliang

$4 \mathrm{Zhu}^{4 \#}$, Lihong Jiang ${ }^{1 \#}$

$5{ }^{1}$ Department of Geriatrics, the Second Affiliated Hospital of Harbin Medical University, Harbin, China

6 2Department of Orthopedic Surgery, the Fourth Affiliated Hospital of Harbin Medical University, Harbin, China

7 3epartment of Nursing, the Second Affiliated Hospital of Harbin Medical University, Harbin, China

$8{ }^{4}$ Institute of Clinical Pharmacology, the Second Affiliated Hospital of Harbin Medical University, Harbin, China

9 *These authors contributed equally to this work.

10 \#Corresponding authors: Wenliang Zhu, Phone/Fax: 86-451-8629-7731, E-mail: wenzwl@yeah.net; Lihong

11 Jiang, Phone: 86-451-8629-7273, Fax: 86-451-8810-9297, E-mail: jianglihong2006@163.com.

\section{ABSTRACT}

22 The incidence of osteoporosis is high in postmenopausal women due to altered estrogen levels and continuous 
23 calcium loss that occurs with aging. Recent studies have shown that microRNAs (miRNAs) are involved in the

24 development of osteoporosis. These miRNAs may be used as potential biomarkers to identify women at a high

25 risk for developing the disease. In this study, whole blood samples were collected from 48 postmenopausal

26 Chinese women with osteopenia or osteoporosis and pooled into six groups according to individual T-scores. A

27 miRNA microarray analysis was performed on pooled blood samples to identify potential miRNA biomarkers

28 for postmenopausal osteoporosis. Five miRNAs (miR-130b-3p, -151a-3p, -151b, -194-5p, and -590-5p) were

29 identified in the microarray analysis. These dysregulated miRNAs were subjected to a pathway analysis

30 investigating whether they were involved in regulating osteoporosis-related pathways. Among them, only miR-

31 194-5p was enriched in multiple osteoporosis-related pathways. Enhanced miR-194-5p expression in women

32 with osteoporosis was confirmed by quantitative reverse transcription-polymerase chain reaction analysis. For

33 external validation, a significant correlation between the expression of miR-194-5p and T-scores was found in

34 an independent patient collection comprised of 24 postmenopausal women with normal bone mineral density, 30

35 postmenopausal women with osteopenia, and 32 postmenopausal women with osteoporosis $(p<0.05)$. Taken

36 together, the present findings suggest that miR-194-5p may be a viable miRNA biomarker for postmenopausal

37 osteoporosis.

38 Keywords: postmenopausal osteoporosis; microRNA; biomarker; miR-194

\section{INTRODUCTION}

43 Postmenopausal women have a high incidence of osteoporosis due to simultaneous existence of multiple 
44 independent predisposing factors, such as estrogen deficiency, continuous calcium loss, and aging (Rosen, 2005).

45 Osteoporosis is mainly characterized by an imbalance between excessive bone resorption by osteoclasts and

46 bone formation by osteoblasts (Raisz, 2005). Osteoporosis leads to low bone mineral density (BMD) and

47 decreased bone strength, which leads to increased risk of fragility fractures (Kanis, 2002). Osteoporosis is a

48 major public health concern worldwide, especially in countries with large aging populations, such as China

49 (Feng et al., 2012). In contrast to developing better treatments for patients with osteoporosis, a proactive

50 approach that identifies patients at high risk for developing osteoporosis is recommended to prevent bone loss,

51 especially when the number of patients with osteoporosis is estimated to grow. (Sanders \& Geraci, 2013; Tella

$52 \&$ Gallagher, 2014). This viewpoint has prompted researchers to develop new osteoporotic biomarkers in recent

53 years to identify postmenopausal women who are at high risk for developing osteoporosis (Vasikaran, 2011;

54 Garnero, 2014).

55 MicroRNAs (miRNAs) are a superfamily of small ( 22 nucleotides), single-stranded, non-coding RNAs

56 (Bushati \& Cohen, 2007). Numerous studies have suggested that miRNAs are important regulators of bone

57 metabolism, and that they participate extensively in bone formation and resorption (Lian et al., 2012; Zhao et al.,

58 2014). For instance, miR-2861 was found to play an important role in promoting osteoblast differentiation by

59 targeting histone deacetylase 5, whereas suppression of miR-2861 led to primary osteoporosis (Li et al., 2009).

60 In addition, a critical miRNA regulator of osteoclast differentiation, miR-223, was also identified (Sugatani \&

61 Hruska, 2007). Because of its implications in osteoclast differentiation, miR-223 may be a promising therapeutic

62 target for correcting excessive osteoclast-mediated bone resorption, which contributes to the pathological

63 development of osteoporosis (Raisz, 2005).

64 Substantial evidence has shown that circulating miRNAs can be used as potential biomarkers for various 
65 human diseases (Mitchell et al., 2008; Häusler et al., 2010; Taurino et al., 2010; Ries et al., 2014). Just recently,

66 studies suggested that three miRNAs, miR-21, miR-133a, and miR-422a, could be feasible biomarkers for

67 postmenopausal osteoporosis (Wang et al., 2012; Cao et al., 2014; Li et al., 2014). In this study, we aimed to

68 investigate whether other potential miRNA biomarkers exist. To do this, we conducted a microarray-based

69 miRNA analysis in whole blood to search for circulating miRNAs with significantly altered peripheral blood

70 expression profiles in postmenopausal osteoporotic women compared to postmenopausal women with

71 osteopenia. T-score-based patient subgrouping was designed to identify miRNA biomarkers with altered

72 expression profiles in peripheral blood that were correlated with a decline in BMD. A systematic pathway

73 analysis was also performed using the Kyoto Encyclopedia of Genes and Genomes (KEGG) (Kanehisa et al.,

74 2006), to reveal whether functional association exists between potential miRNA biomarkers and osteoporosis-

75 related pathways. Lastly, quantitative reverse transcription-polymerase chain reaction (qRT-PCR) was used to

76 validate independently the potential biomarkers identified from the initial screen. In summary, our efforts aim to

77 provide new miRNA biomarkers as tools allowing for better diagnosis and prevention of postmenopausal

78 osteoporosis in the future.

79 MATERIALS AND METHODS

80 Participant characteristics

81 This study was approved by the Ethics Committee of Harbin Medical University (Approval number: 2014-R-

82 020). Each participant enrolled in this study was informed of the project and had signed a written consent.

83 Twenty-three postmenopausal Chinese women with osteopenia (moderate decrease in BMD, spine T-score $\leqslant$ -

841.0 and $>-2.5$ ) and 25 postmenopausal Chinese women with osteoporosis (marked decline in BMD, spine T-

85 score $\leqslant-2.5)$ were recruited and further divided into six subgroups according to their T-score measurements 
86 (Table 1). Dividing the patients into six subgroups allowed investigation of whether altered expression of a 87 particular miRNA may be correlated with bone loss during pathological progression from osteopenia to 88 osteoporosis. Both the spine and femoral neck T-score was measured for each participant. In addition, an 89 independent collection of participants was recruited for the purpose of external validation. This group was 90 comprised of 24 postmenopausal women with a normal BMD, 30 postmenopausal women with osteopenia, and

9132 postmenopausal women with osteoporosis (Table S1). Participants were given a questionnaire to obtain their 92 medication history, and a hospital examination verified that no serious complications, such as cancer, Type II 93 diabetes, and/or cardiac disease were present. All the participants ranged in age from 59 to 70 and had been 94 postmenopausal for at least 12 months (no menses 12 months after the date of the last menses). T-scores of the 95 spine (L1-L4) and femoral neck were obtained using a dual energy X-ray absorptiometry scanner (Hologic Inc.,

\section{Bedford, MA).}

\section{Blood sample collection and total RNA extraction}

98 Five milliliters of whole blood was obtained from each participant. Each whole blood sample was independently

99 lysed using Red Blood Cell (RBC) Lysis Solution (Beyotime, Shanghai, China) and centrifuged for 10 min at $100450 \times g$. TRIzol reagent (Invitrogen, Shanghai, China) was used to extract RNA from the precipitate. RNA 101 extraction was completed within 30 min after blood collection from each participant. Isolated RNA eluate was stored at $-80^{\circ} \mathrm{C}$. After blood sample collection was complete, all RNA extraction samples from individual participants were thawed and pooled separately into six subgroups corresponding to the spine T-score values

104 (Table 1). The pooled samples were then stored at $-80^{\circ} \mathrm{C}$ for future experiments. The same procedure was applied to the whole blood samples for external validation (Table S1); however, these RNA samples were not pooled. 
107

108

109

110

111

112

113

114

115

116

117

118

119

120

121

122

123

124

125

126

127

\section{Microarray scanning}

An Agilent Human miRNA microarray (Release 19.0, $8 \times 60 \mathrm{~K}$ ) was used for global scanning of miRNA expression in pooled RNA samples. Sample labeling, microarray hybridization, and washing were performed based on the manufacturer's standard protocols (Agilent Technologies Inc., Santa Clara, CA). Briefly, total RNA was dephosphorylated, denatured, and then labeled with Cyanine-3-CTP. After purification, labeled RNAs were hybridized onto the microarray. After washing, the arrays were scanned with an Agilent Scanner G2505C (Agilent Technologies Inc.). Feature Extraction software (version 10.7.1.1, Agilent Technologies Inc.) was used to analyze microarray images and obtain raw data. Next, GeneSpring software (version 12.5; Agilent Technologies Inc.) was used to complete the basic analysis using raw data. The raw data was normalized with the quantile algorithm. If the probes with a positive normalized expression value were flagged as "Detected" in at least $100 \%$ of samples, they were chosen for further analysis. Differentially expressed miRNAs were then identified through fold change as well as the $p$ value calculated using a Student's $t$-test. The threshold set for significantly up- and down-regulated genes was a fold change $>2.0$ and a $p$ value $<0.05$. The miRNA microarray assay was performed by Shanghai OEBiotech Technology Co, Ltd. (Shanghai, China). Microarray scanning data have been submitted to the Gene Expression Omnibus with an accession number GSE64433.

\section{qRT-PCR analysis}

To avoid false positives during microarray detection, a qRT-PCR assay was used to validate further the significantly dysregulated miRNAs identified in the pooled RNA extraction samples. RNA eluate $(0.4 \mu \mathrm{L})$ was reverse transcribed into cDNA in a $10-\mu \mathrm{L}$ reaction volume, using a high-capacity cDNA reverse transcription kit (Applied Biosystems, Foster City, CA). Briefly, $1.0 \mu \mathrm{L} 10 \times \mathrm{RT}$ Buffer, $0.4 \mu \mathrm{L} 25 \times \mathrm{dNTP}$ Mix (100 mM), $1.0 \mu \mathrm{L}$ $100 \mathrm{mM}$ RT miRNA primers, $0.5 \mu \mathrm{L}$ MultiScribe ${ }^{\mathrm{TM}}$ Reverse Transcriptase, and $0.5 \mu \mathrm{g}$ RNA were mixed, and 


\section{Pathway analysis}

144 The HuGE Navigator Gene Prospector online tool was used to search for literature-reported osteoporosis-related 
149 was considered statistically significant only if the Bonferroni-adjusted $p<0.05$ (Bland \& Altman, 1995).

150 An integrative retrieval from two miRNA-target interaction (MTI) databases, including miRSel (Naeem et al.,

151 2010) and miRTarBase (Hsu et al., 2014), was applied to search for experimentally validated target genes of six

152 miRNAs, including miR-130b-3p, -133a, -151a-3p, -151b, -194-5p, and -590-5p in humans. After uploading the

153 official symbols of the target genes of each miRNA onto the DAVID website, the online functional annotation

154 tool was used to reveal whether these target genes were involved in osteoporosis-related KEGG pathways.

\section{Statistical analysis}

156 All data are expressed as the mean \pm SD. Statistical analysis was performed using Student's $t$-test or a Pearson 157 correlation test. GraphPad Prism v6.0 was used to conduct statistical analysis. Differences were considered as 158 statistically significant when $p<0.05$.

\section{RESULTS}

Microarray scanning identified six miRNAs with increased peripheral blood expression in participants

162 To search for potential miRNA biomarkers for postmenopausal osteoporosis, a comprehensive miRNA expression analysis was performed on pooled RNA samples isolated from postmenopausal Chinese women with 


\section{osteoporosis}

172 Due to the high false positive rate of the microarray method, we validated the circulating blood expression levels

173 of the six significant microarray-identified miRNAs by using qRT-PCR. Except for miR-660-5p, all of the

174 miRNAs tested showed remarkably higher expression in the peripheral blood of patients with osteoporosis

175 compared to that observed in the blood of patients with osteopenia $(p<0.05$, Fig. 2$)$. Furthermore, we

176 investigated whether a significant linear correlation existed between miRNA expression and spine T-score. Our

177 results showed that the expression of four miRNAs, including miR-130b-3p, -151a-3p, -151b, -194-5p, in

178 circulating blood were significantly and negatively correlated with a decline in $\operatorname{BMD}(p<0.05$, Fig. S1). Of note,

179 the expression levels of miR-151b and $-194-5 p$ were also significantly and negatively correlated with femoral

180 neck T-scores $(p<0.05$, Fig. S1). This finding suggested that these two miRNAs might be more suitable for

181 further consideration as potential biomarkers for postmenopausal osteoporosis.

\section{miR-194-5p is implicated in multiple osteoporosis-related pathways}

183 To explore a potential functional association between the miRNAs identified in this study and osteoporosis, the

online bioinformatics tool DAVID was used to investigate whether the identified miRNAs regulate osteoporosis-

related pathways by targeting the mRNA genes in each pathway. There were 985 genes reported in the literature

to be functionally associated with osteoporosis. Analysis of their official gene symbols with the DAVID website

revealed 19 KEGG pathways with over-represented osteoporosis-related genes (Table 2). These pathways were 
191 133a,which was previously suggested as a feasible biomarker for postmenopausal osteoporosis (Wang et al.,

192 2012; Li et al., 2014). We then investigated involvement of each miRNAs in regulating osteoporosis-related

193 pathways. MiR-151b was found to lack experimentally-validated MTIs; therefore, it was excluded from further

194 analysis. The remaining four miRNAs and miR-133a, the known osteoporosis biomarker, underwent subsequent

195 pathway analysis. As expected, miR-133a was implicated in six osteoporosis-related pathways such as cytokine-

196 cytokine receptor interaction (Table 2). This finding was consistent with the previous study, in which the

197 authors demonstrated that miR-133a targeted multiple pathway-associated genes, such as chemokine (C-X-C

198 motif) ligand 11 (CXCL11) and chemokine (C-C motif) receptor 3 (CXCR3) (Wang et al., 2012). In our analysis,

199 we determined that miR-194-5p was functionally associated with eight osteoporosis-related pathways. This

200 result implies that by targeting many genes, miR-194-5p may have effects on the TGF-beta and Wnt signaling

201 pathways, which were shown to play critical roles in the pathology of postmenopausal osteoporosis (Krishnan,

202 Bryant \& Macdougald, 2006; Nistala et al., 2010).

203 External validation confirmed increased expression of miR-194-5p in postmenopausal osteoporosis

204 External validation of miR-194-5p expression in the blood circulation of postmenopausal women with

205 osteoporosis was performed by qRT-PCR analysis. To do this, an independent collection of participants was

206 recruited, which was comprised of 24 postmenopausal women with normal BMD, 30 postmenopausal women

207 with osteopenia, and 32 postmenopausal women with osteoporosis (Table S1). A significant increase in

208 peripheral expression of miR-194-5p was found in postmenopausal women with osteopenia or osteoporosis,

209 compared to postmenopausal women with normal BMD $(p<0.001$, Fig. 3A). A remarkable negative correlation

210 was also found between miR-194-5p expression and T-scores $(p<0.05$, Fig. 3B and C). Moreover, the qRT-

211 PCR method established in this study efficiently detected miR-194-5p expression in peripheral blood with high 
212 specificity (Fig. S2), thus increasing our confidence in the reliability of the external validation results.

\section{DISCUSSION}

214 Osteoporosis is an independent factor that increases the risk of fragility fractures in postmenopausal women

215 (Kanterewicz et al., 2014). In this study, a microarray-based scanning approach followed by qRT-PCR

216 validation and pathway analysis was conducted to identify potential circulating miRNA biomarkers for

217 postmenopausal osteoporosis. Our study revealed that miR-194-5p should be considered a potential biomarker

218 for postmenopausal osteoporosis, in addition to three previously recognized miRNAs, miR-21, $-133 \mathrm{a}$ and $-422 \mathrm{a}$

219 (Wang et al., 2012; Cao et al., 2014; Li et al., 2014).

220 A miRNA microarray technique was used in this study for high-throughput detection of hundreds of miRNAs

221 at a relatively low cost compared to gene sequencing. The success of this method in identifying potential

miRNA biomarkers has been widely confirmed in many studies on various diseases (Mitchell et al., 2008;

223 Häusler et al., 2010; Taurino et al., 2010; Ries et al., 2014). However, a major pitfall of this approach is a high

224 false positive rate. Thus, qRT-PCR is generally performed to validate significant miRNAs identified by

225 microarray. In contrast to previous studies (Wang et al., 2012; Cao et al., 2014; Li et al., 2014), we used pooled

226 RNA samples according to T-score measurements, rather than those of individual participants. This

227 experimental design aims to minimize individual, physical differences to reveal potential relationships between

228 miRNA expression and a decline in BMD. Indeed, this approach led to the identification of six dysregulated

229 miRNAs by microarray analysis. Among them, elevated expression miR-151b and miR-194-5p was validated in

230 the blood of patients with osteoporosis, and we determined a remarkable negative correlation with enhanced

231 miR-194-5p expression and bone loss in both the spine and femoral neck by qRT-PCR analysis. However, these

232 results should be taken with caution because the sample size of each T-score subgroup was small. In the case of 
233

234

235

236

238

240

241

242

243

244

245

246

247

248

249

250
miR-194-5p expression, we confirmed its negative correlation with the decline of BMD in a larger independent population including 86 subjects covering a wider BMD range. This finding suggested that miR-194-5p could be a potential miRNA biomarker for osteoporosis because its peripheral expression is associated with pathological bone loss in postmenopausal women.

To further investigate potential functional roles of these significant miRNAs in osteoporosis, an evidencebased pathway analysis was performed on target genes of miRNAs and osteoporosis-related genes at the pathway level using KEGG, a reference knowledge base of signaling pathways in humans (Kanehisa et al., 2006). Experimental and literature-reported evidence for MTIs ((Naeem et al., 2010; Hsu et al., 2014) and geneosteoporosis association ( $\mathrm{Yu}$ et al., 2008) increased our trust in the analysis results. There were nearly 1000 genes reported in the literature that were related to osteoporosis, suggesting complex pathological mechanisms underlying this disease (Raisz, 2005; Rachner, Khosla S \& Hofbauer, 2011). In total, 19 KEGG pathways were found to be significantly associated with osteoporosis (Bonferroni-adjusted $p<0.05$ ). Of note, approximately $9 \%$ of osteoporosis-related genes were involved in the cytokine-cytokine receptor interaction pathway, indicating critical roles for cytokines and their corresponding receptors in the pathogenesis of osteoporosis (Manolagas and Jilka, 1995). This result supported previous findings obtained from a bibliometric network (Sun et al., 2013). Although different data resources were used, Sun and colleagues (2013) also observed that cytokine-cytokine receptor interaction might be the most osteoporosis-related-genes-enriched KEGG pathway. Obvious gene enrichment in the Wnt signaling pathway was also determined by our pathway analysis. This finding was in agreement with the notion that Wnt antagonists should be further developed for osteoporosis treatment (Rawadi \& Roman-Roman, 2005; Chan A, van Bezooijen \& Löwik, 2007).

MiRNA can influence a signaling pathway by targeting many pathway-related genes (Kowarsch, 2011). Our 
254 analysis highlighted miR-194-5p as a potential biomarker for osteoporosis, as it was found to target genes in

255 eight osteoporosis-related pathways. It was not surprising that miR-133a, a validated biomarker for osteoporosis

256 (Wang et al., 2012; Li et al., 2014), targeted genes in six osteoporosis-related pathways. Based on our previous

257 studies of multi-pathway renoprotectants (Xu et al., 2014), enriched regulation of multiple pathways associated

258 with osteoporosis may suggest a potential functional role for miR-194-5p in the pathogenesis of osteoporosis.

259 Just recently, it was confirmed that by targeting chicken ovalbumin upstream promoter-transcription factor II

260 (COUP-TFII), miR-194-5p stimulated osteogenesis and inhibited adipogenesis if it was specifically

261 overexpressed in mesenchymal stem cells (Jeong et al., 2014). COUP-TFII was sought as a critical regulator of

262 mesenchymal stem cell fate, regulating stem cell differentiation into osteoblasts and adipocytes. This

263 observation suggested that miR-194-5p might play an important role in osteoblast differentiation. Furthermore,

264 modulation of Wnt signaling was implicated in COUP-TFII-mediated regulation on mesenchymal cell

265 commitment and differentiation (Xie et al., 2011). The results of our pathway analysis are in line with those of

266 this report, as we determined that miR-194-5p might influence Wnt signaling by targeting genes in the Wnt

267 signaling pathway. Further functional experiments are needed to validate these results. Recently, it was

268 demonstrated that both elevated bone resorption and stimulated bone formation could be simultaneously

269 observed in an ovariectomy model, despite uncoupling between excessive bone resorption by osteoclasts and

270 bone formation by osteoblasts that eventually led to a net bone loss (Matsuoka et al., 2014). Taken together, we

271 propose that the above evidences may reasonably explain the findings observed in our study where miR-194-5p

272 showed elevated expression the peripheral blood of postmenopausal women with osteoporosis and, at the same

273 time, a negative correlation between its expression and the extent of bone loss.

274 In conclusion, we integrated microarray-based miRNA scanning, qRT-PCR, and pathway analysis to identify 
275 potential miRNA biomarkers for postmenopausal osteoporosis. A limitation of our study was that only 331

276 miRNAs were detected by miRNA microarray. Despite this, we were able to identify miR-194-5p as a potential 277 biomarker for postmenopausal osteoporosis. We confirmed that miR-194-5p expression was elevated in the 278 peripheral blood of postmenopausal osteoporotic women. Furthermore, miR-194-5p was negatively correlated 279 with the decline of BMD in the spine and femoral neck, and it was found to be associated with multiple 280 osteoporosis-related pathways. Nevertheless, further research in a larger study population will be necessary to 281 validate our findings.

282

283

REFERENCES

284 Bland JM, Altman DG. 1995. Multiple significance tests: the Bonferroni method. British medical 285 journal, 310(6973): 170

286 Bushati N, Cohen SM. 2007. microRNA functions. Annual Review of Cell and Developmental Biology, 23:175287205 DOI 10.1146/annurev.cellbio.23.090506.123406.

288 Cao Z, Moore BT, Wang Y, Peng XH, Lappe JM, Recker RR, Xiao P. 2014. MiR-422a as a potential 289 cellular microRNA biomarker for postmenopausal osteoporosis. PLoS One, 9(5):e97098 DOI 290 10.1371/journal.pone.0097098.

291 Chan A, van Bezooijen RL, Löwik CW. 2007. A new paradigm in the treatment of osteoporosis: Wnt pathway 292 proteins and their antagonists. Current opinion in investigational drugs (London, England: 2000), 8(4):293-298

293 Feng Z, Liu C, Guan X, Mor V. 2012. China's rapidly aging population creates policy challenges in shaping a 294 viable long-term care system. Health Affairs, 31(12):2764-2773 DOI 10.1377/hlthaff.2012.0535.

295 Garnero P. 2014. New developments in biological markers of bone metabolism in osteoporosis. Bone, 66:46-55 
DOI 10.1016/j.bone.2014.05.016.

Häusler SF, Keller A, Chandran PA, Ziegler K, Zipp K, Heuer S, Krockenberger M, Engel JB, Hönig A,

Scheffler M, Dietl J, Wischhusen J. 2010. Whole blood-derived miRNA profiles as potential new tools for ovarian cancer screening. British journal of cancer, 103(5):693-700 DOI 10.1038/sj.bjc.6605833.

\section{Hsu SD, Tseng YT, Shrestha S, Lin YL, Khaleel A, Chou CH, Chu CF, Huang HY, Lin CM, Ho SY, Jian}

TY, Lin FM, Chang TH, Weng SL, Liao KW, Liao IE, Liu CC, Huang HD. 2014. miRTarBase update 2014:

an information resource for experimentally validated miRNA-target interactions. Nucleic acids research, 42(Database issue):D78-D85 DOI 10.1093/nar/gkt1266.

Huang da W, Sherman BT, Lempicki RA. 2009. Systematic and integrative analysis of large gene lists using DAVID bioinformatics resources. Nature protocols, 4(1):44-57 DOI 10.1038/nprot.2008.211.

Jeong BC, Kang IH, Hwang YC, Kim SH, Koh JT. 2014. MicroRNA-194 reciprocally stimulates osteogenesis and inhibits adipogenesis via regulating COUP-TFII expression. Cell Death and Disease, 5:e1532 DOI 10.1038/cddis.2014.485.

Kanehisa M, Goto S, Hattori M, Aoki-Kinoshita KF, Itoh M, Kawashima S, Katayama T, Araki M, Hirakawa M. 2006. From genomics to chemical genomics: new developments in KEGG. Nucleic acids research, 34(Database issue):D354-D357 DOI 10.1093/nar/gkj102.

Kanis JA. 2002. Diagnosis of osteoporosis and assessment of fracture risk. The Lancet, 359(9321):1929-1936 DOI 10.1016/S0140-6736(02)08761-5.

\section{Kanterewicz E, Puigoriol E, García-Barrionuevo J, del Rio L, Casellas M, Peris P; Frodos Research}

Group. 2014. Prevalence of vertebral fractures and minor vertebral deformities evaluated by DXA-assisted vertebral fracture assessment (VFA) in a population-based study of postmenopausal women: the FRODOS study. 
318 Kowarsch A, Preusse M, Marr C, Theis FJ. 2011. miTALOS: analyzing the tissue-specific regulation of

319 signaling pathways by human and mouse microRNAs. $R N A, \mathbf{1 7 ( 5 ) : 8 0 9 - 8 1 9 . ~ D O I ~ 1 0 . 1 2 6 1 / r n a . 2 4 7 4 5 1 1 . ~}$

320 Krishnan V, Bryant HU, Macdougald OA. 2006. Regulation of bone mass by Wnt signaling. The Journal of

321 clinical investigation, 116(5):1202-1209 DOI 10.1172/JCI28551.

Li H, Wang Z, Fu Q, Zhang J. 2014. Plasma miRNA levels correlate with sensitivity to bone mineral density

in postmenopausal osteoporosis patients. Biomarkers, 19(7):553-556 DOI 10.3109/1354750X.2014.935957.

Li H, Xie H, Liu W, Hu R, Huang B, Tan YF, Xu K, Sheng ZF, Zhou HD, Wu XP, Luo XH. 2009. A novel

Lian JB, Stein GS, Van Wijnen AJ, Stein JL, Hassan MQ, Gaur T, Zhang Y. 2012. MicroRNA control of

Manolagas SC, Jilka RL. 1995. Bone marrow, cytokines, and bone remodeling. Emerging insights into the

Matsuoka K, Park KA, Ito M, Ikeda K, Takeshita S. 2014. Osteoclast-derived complement component 3a stimulates osteoblast differentiation. Journal of Bone and Mineral Research, 29(7):1522-1530 DOI 
338 based markers for cancer detection. Proceedings of the National Academy of Sciences of the United States of 339 America, 105(30):10513-10518 DOI 10.1073/pnas.0804549105.

340 Naeem H, Küffner R, Csaba G, Zimmer R. 2010. miRSel: automated extraction of associations between

341 microRNAs and genes from the biomedical literature. BMC Bioinformatics, 11:135 DOI 10.1186/1471-2105-11342135.

343 Nistala H, Lee-Arteaga S, Siciliano G, Smaldone S, Ramirez F. 2010. Extracellular regulation of 344 transforming growth factor $\beta$ and bone morphogenetic protein signaling in bone. Annals of the New York 345 Academy of Sciences, 1192(1):253-256 DOI 10.1111/j.1749-6632.2009.05350.x.

346 Rachner TD, Khosla S, Hofbauer LC. 2011. Osteoporosis: now and the future. The Lancet, 377(9773):1276-

347

348

349

350

351

352

353

354

355

356

357

358

1287 DOI 10.1016/S0140-6736(10)62349-5.

Raisz LG. 2005. Pathogenesis of osteoporosis: concepts, conflicts, and prospects. The Journal of clinical investigation, 115(12):3318-3325 DOI 10.1172/JCI27071.

Rawadi G, Roman-Roman S. 2005. Wnt signalling pathway: a new target for the treatment of osteoporosis. Expert Opinion on Therapeutic Targets, 9(5):1063-1077 DOI 10.1517/14728222.9.5.1063.

Ries J, Vairaktaris E, Agaimy A, Kintopp R, Baran C, Neukam FW, Nkenke E. 2014. miR-186, miR-3651 and miR-494: potential biomarkers for oral squamous cell carcinoma extracted from whole blood. Oncology reports, 31(3):1429-1436 DOI 10.3892/or.2014.2983.

Rosen CJ. 2005. Postmenopausal osteoporosis. New England Journal of Medicine, 353(6):595-603 DOI 10.1056/NEJMcp043801.

Sanders S, Geraci SA. 2013. Osteoporosis in postmenopausal women: considerations in prevention and treatment:(women's health series). Southern medical journal, 106(12):698-706 DOI 
10.1097/SMJ.0b013e3182a0df8b.

360 Sugatani T, Hruska KA. 2007. MicroRNA-223 is a key factor in osteoclast differentiation. Journal of cellular

361 biochemistry, 101(4):996-999 DOI 10.1002/jcb.21335.

362 Taurino C, Miller WH, McBride MW, McClure JD, Khanin R, Moreno MU, Dymott JA, Delles C,

363 Dominiczak AF. 2010. Gene expression profiling in whole blood of patients with coronary artery disease.

364 Clinical science, 119(8):335-343 DOI 10.1042/CS20100043.

365 Sun GJ, Guo T, Chen Y, Xu B, Guo JH, Zhao JN. 2013. Significant pathways detection in Osteoporosis based on the bibliometric network. European Review for Medical and Pharmacological Sciences, 17(1):1-7

Tella SH, Gallagher JC. 2014. Prevention and treatment of postmenopausal osteoporosis. The Journal of steroid biochemistry and molecular biology, 142:155-170 DOI 10.1016/j.jsbmb.2013.09.008.

Group. 2011. Markers of bone turnover for the prediction of fracture risk and monitoring of osteoporosis

377 Xie X, Qin J, Lin SH, Tsai SY, Tsai MJ. 2011. Nuclear receptor chicken ovalbumin upstream promoter-

378 transcription factor II (COUP-TFII) modulates mesenchymal cell commitment and differentiation. Proceedings

379 of the National Academy of Sciences of the United States of America, 108(36):14843-14848 DOI 
$380 \quad 10.1073 /$ pnas.1110236108.

381 Xu J, Meng K, Zhang R, Yang H, Liao C, Zhu W, Jiao J. 2014. The use of functional chemical-protein 382 associations to identify multi-pathway renoprotectants. PLoS One, 9(5):e97906 DOI 383 10.1371/journal.pone.0097906.

384 Yu W, Wulf A, Liu T, Khoury MJ, Gwinn M. 2008. Gene Prospector: an evidence gateway for evaluating 385 potential susceptibility genes and interacting risk factors for human diseases. BMC Bioinformatics, 9:528 DOI $386 \quad 10.1186 / 1471-2105-9-528$.

387 Zhao X, Xu D, Li Y, Zhang J, Liu T, Ji Y, Wang J, Zhou G, Xie X. 2014. MicroRnAs regulate bone 388 metabolism. Journal of bone and mineral metabolism, 32(3):221-231 DOI 10.1007/s00774-013-0537-7. 


\section{FIGURE LEGENDS}

403 Fig. 1. Microarray scanning identified six significantly upregulated miRNAs in samples obtained from patients

404 with postmenopausal osteoporosis. Statistical comparison was performed between participants with osteopenia 405 (S1-S3) and osteoporosis (S4-S6) using a Student's $t$-test.

406 Fig. 2. qRT-PCR validation results $(n=4)$. Statistical comparison was performed between participants with 407 osteopenia (S1-S3) and those with osteoporosis (S4-S6), using a Student's $t$-test.

408 Fig. 3. Results of external validation by qRT-PCR analysis. An obvious increase in miR-194-5p expression was 409 observed in participants with osteopenia or osteoporosis compared with participants that had a normal BMD $(n=$ 410 86) (A). There was no significant difference in age across he groups. A significant correlation was found 411 between miR-194-5p expression and spine (B) and femoral neck T-scores (C).

\section{SUPPLEMENTAL INFORMATION}

413 Table S1. External validation study participant information.

414 Table S2. Human gene sequences involved in this study.

415 Table S3. Microarray scanning results.

416 Fig. S1. Correlation between mean miRNA expression and mean T-score (spine T-score or femoral neck T-score, $417 \mathrm{n}=6)$. Mean miRNA expression is the mean measured value $(\mathrm{n}=4)$ of each pooled RNA sample by qRT-PCR; 418 mean T-score is the average T-score of the participants in the corresponding subgroup.

419 Fig. S2. Evaluation method for miR-194-5p detection using qRT-PCR. A linear relationship was observed between the negative logarithmic value of the concentration of synthetic miR-194-5p and the CT value detected by qRT-PCR $\left(\mathrm{R}^{2}=0.990\right)$. This experiment was repeated three times. 


\section{1}

Fig. 1

Fig. 1. Microarray scanning identified six significantly upregulated miRNAs in samples obtained from patients with postmenopausal osteoporosis. Statistical comparison was performed between participants with osteopenia (S1-S3) and osteoporosis (S4-S6) using a Student's t-test.

miR-130b-3p

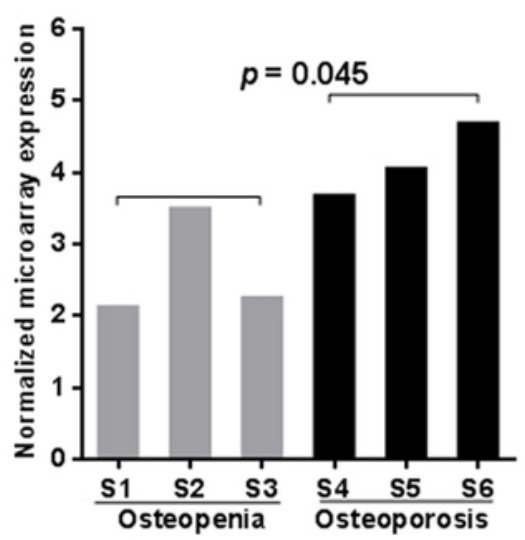

miR-194-5p

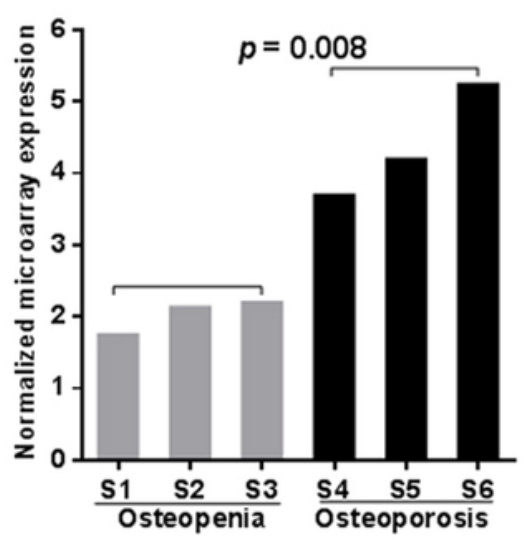

miR-151a-3p

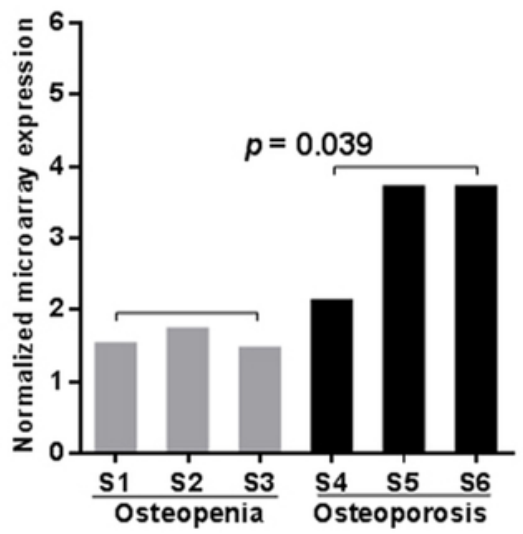

$\operatorname{miR}-590-5 p$

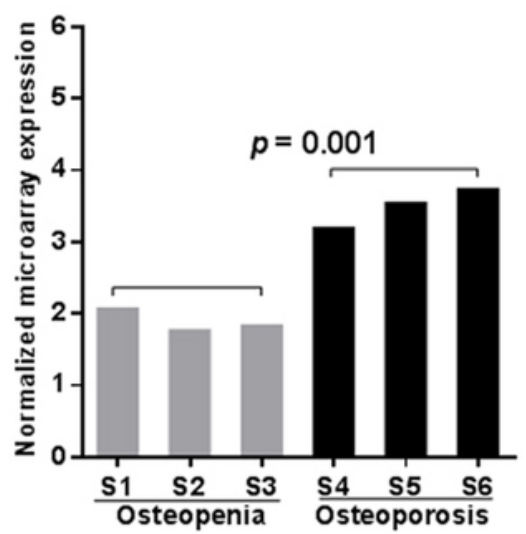

$\operatorname{miR}-151 b$

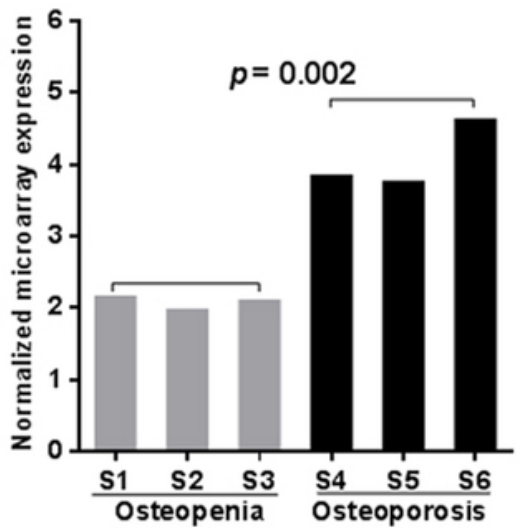

$\operatorname{miR}-660-5 p$

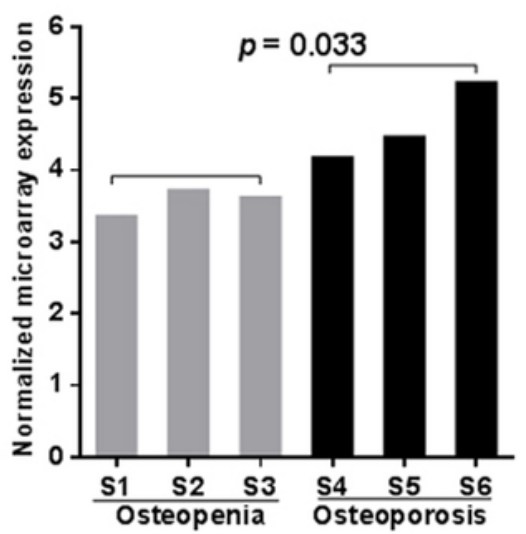




\section{2}

Fig. 2

Fig. 2. $q R T-P C R$ validation results $(n=4)$. Statistical comparison was performed between participants with osteopenia (S1-S3) and those with osteoporosis (S4-S6), using a Student's t-test.

miR-130b-3p

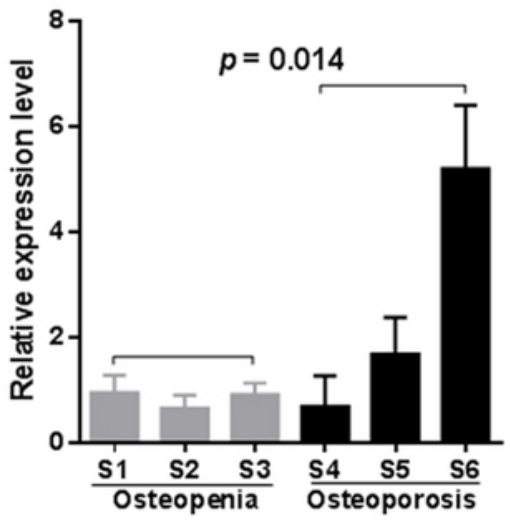

miR-194-5p

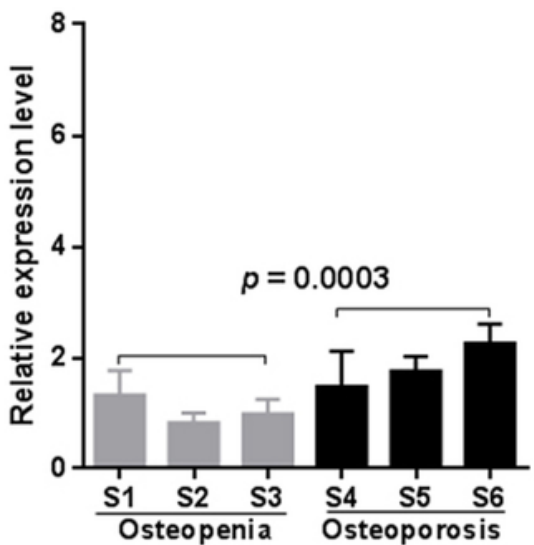

miR-151a-3p

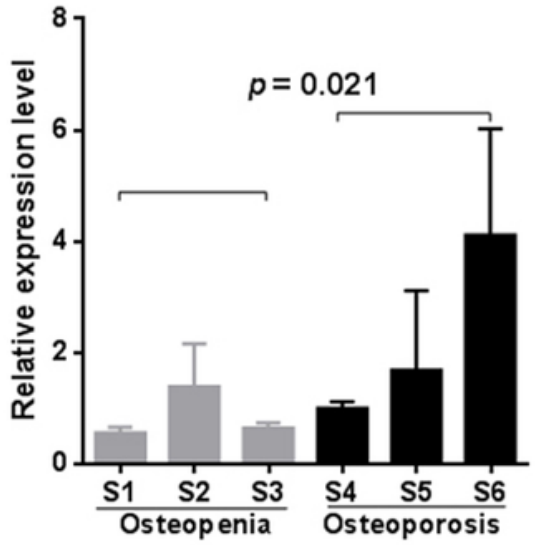

miR-590-5p

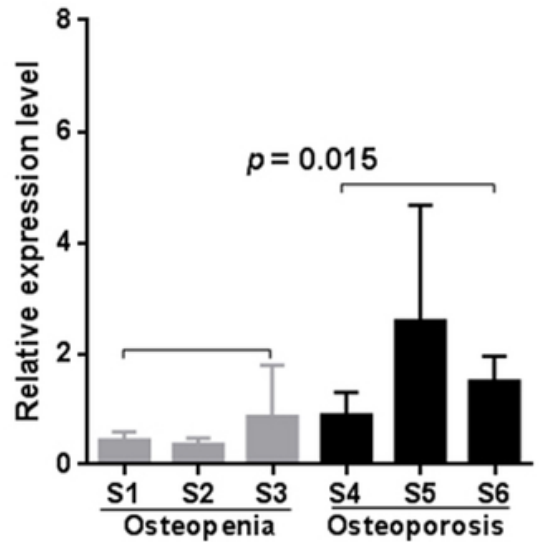

miR-151b

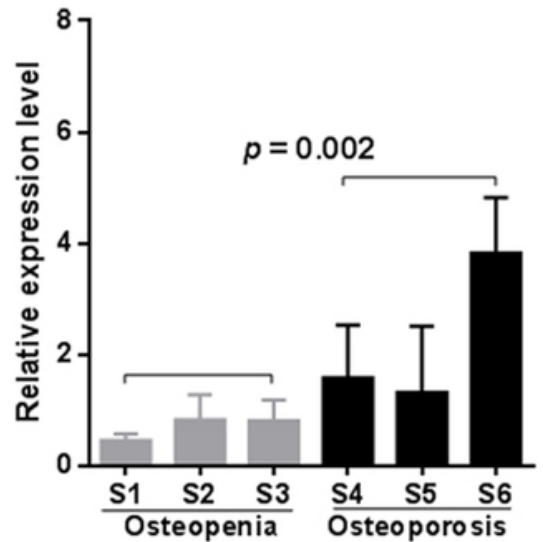

miR-660-5p

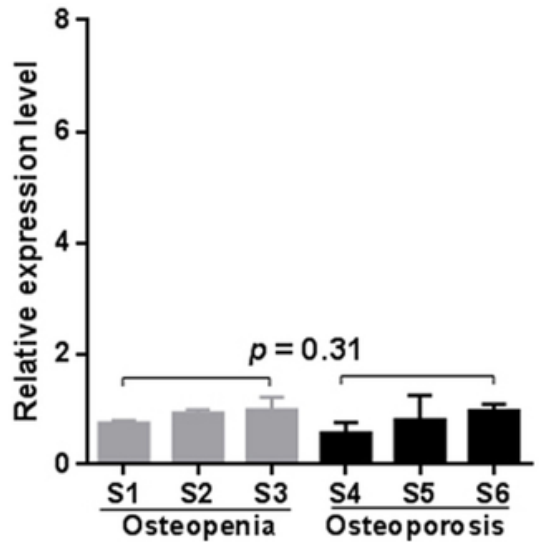




\section{3}

Fig. 3

Fig. 3. Results of external validation by $q R T-P C R$ analysis. An obvious increase in miR-194-5p expression was observed in participants with osteopenia or osteoporosis compared with participants that had a normal BMD $(n=86)(\mathbf{A})$. There was no significant difference in age across he groups. A significant correlation was found between miR-194-5p expression and spine (B) and femoral neck T-scores (C).

A

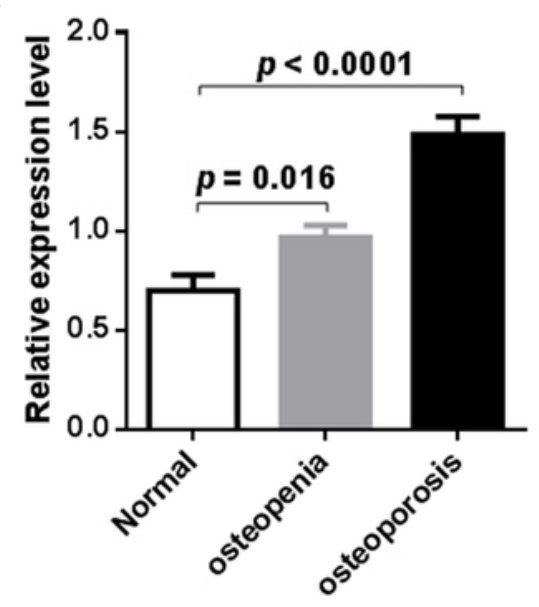

B

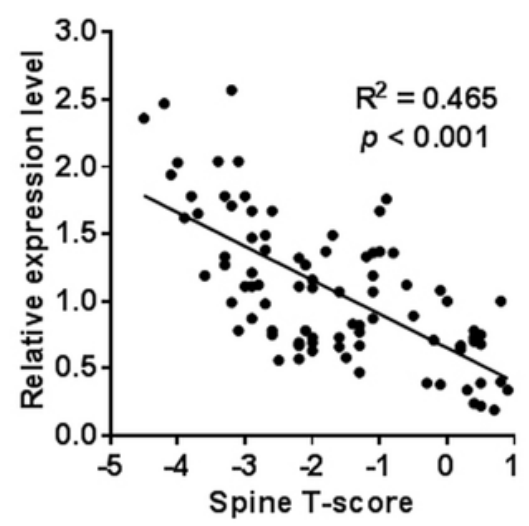

C

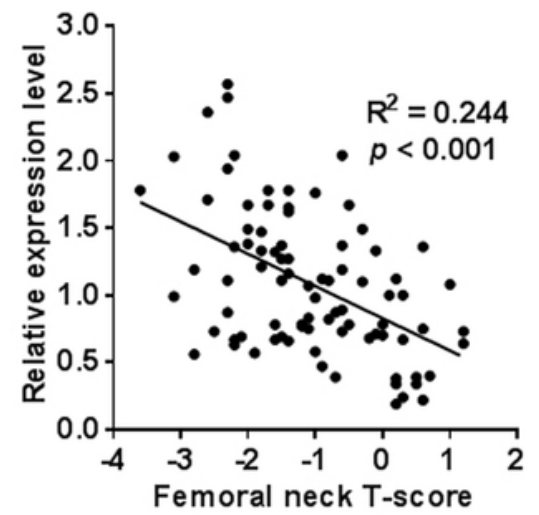




\section{Table $\mathbf{1}$ (on next page)}

Table 1

Table 1. Characteristics of participants recruited for the microarray study. 
2 Table 1. Characteristics of participants recruited for the microarray study.

\begin{tabular}{|l|l|l|l|l|}
\hline Disease $(\mathrm{n})$ & Subgroup $(\mathrm{n})$ & Age $($ year $)$ & Spine T-score & Femoral neck T-score \\
\hline Osteopenia $(\mathrm{n}=23)$ & $\mathrm{S} 1,-1.5 \leqslant$ Spine T-score $\leqslant-1.0(\mathrm{n}=7)$ & $63.1 \pm 2.4$ & $-1.21 \pm 0.20$ & $-0.99 \pm 0.63$ \\
\hline & $\mathrm{S} 2,-2.0 \leqslant$ Spine T-score $<-1.5(\mathrm{n}=7)$ & $66.4 \pm 3.4$ & $-1.84 \pm 0.18$ & $-1.24 \pm 1.03$ \\
\hline & $\mathrm{S} 3,-2.5<$ Spine T-score $<-2.0(\mathrm{n}=9)$ & $64.8 \pm 3.7$ & $-2.19 \pm 0.12$ & $-1.31 \pm 0.60$ \\
\hline Osteoporosis $(\mathrm{n}=25)$ & $\mathrm{S} 4,-3.0 \leqslant$ Spine T-score $\leqslant-2.5(\mathrm{n}=$ & $66.3 \pm 3.6$ & $-2.79 \pm 0.14$ & $-2.27 \pm 0.76$ \\
\hline & $10)$ & & & $-2.24 \pm 0.69$ \\
\hline & $\mathrm{S} 5,-4.0 \leqslant$ Spine T-score $<-3.0(\mathrm{n}=9)$ & $64.6 \pm 3.5$ & $-3.53 \pm 0.28$ & $-2.77 \pm 0.54$ \\
\hline
\end{tabular}

3 Data are expressed as the mean \pm SD. There was no significant age difference across participant subgroups. 


\section{Table 2 (on next page)}

Table 2

Table 2. Results of KEGG pathway analysis. 
2 Table 2. Results of KEGG pathway analysis.

\begin{tabular}{|c|c|c|}
\hline KEGG pathway & Count (Adjusted $p$-value) & miRNA (n) \\
\hline Cytokine-cytokine receptor interaction & $84(1.46 \mathrm{E}-17)$ & miR-133a (6) \\
\hline Adipocytokine signaling pathway & $38(2.84 \mathrm{E}-16)$ & - \\
\hline TGF-beta signaling pathway & $38(1.70 \mathrm{E}-11)$ & $\begin{array}{l}\operatorname{miR}-130 b-3 p(4), \text { miR-194-5p }\left(6^{a}\right), \text { miR-590- } \\
5 p(3)\end{array}$ \\
\hline Wnt signaling pathway & $50(3.81 \mathrm{E}-10)$ & $\operatorname{miR}-194-5 p\left(7^{a}\right)$ \\
\hline Steroid hormone biosynthesis & $25(1.90 \mathrm{E}-9)$ & - \\
\hline MAPK signaling pathway & $69(6.66 \mathrm{E}-9)$ & miR-133a (7); miR-194-5p (6) \\
\hline Apoptosis & $33(7.94 \mathrm{E}-8)$ & miR-133a (4); miR-194-5p (3) \\
\hline Hedgehog signaling pathway & $25(3.42 \mathrm{E}-7)$ & - \\
\hline Toll-like receptor signaling pathway & $33(5.50 \mathrm{E}-6)$ & miR-194-5p (4) \\
\hline Jak-STAT signaling pathway & $41(6.06 \mathrm{E}-5)$ & miR-133a (6); miR-194-5p (6) \\
\hline Hematopoietic cell lineage & $28(8.20 \mathrm{E}-5)$ & - \\
\hline Neurotrophin signaling pathway & $35(1.02 \mathrm{E}-4)$ & miR-133a (4); miR-194-5p (4) \\
\hline Androgen and estrogen metabolism & $16(9.00 \mathrm{E}-4)$ & - \\
\hline NOD-like receptor signaling pathway & $21(1.61 \mathrm{E}-3)$ & - \\
\hline $\mathrm{T}$ cell receptor signaling pathway & $28(9.15 \mathrm{E}-3)$ & miR-133a (4); miR-194-5p (3) \\
\hline Neuroactive ligand-receptor interaction & $51(1.26 \mathrm{E}-02)$ & - \\
\hline Complement and coagulation cascades & $20(2.98 \mathrm{E}-2)$ & - \\
\hline PPAR signaling pathway & $20(2.98 \mathrm{E}-2)$ & - \\
\hline RIG-I-like receptor signaling pathway & $20(4.44 \mathrm{E}-02)$ & - \\
\hline
\end{tabular}

3 Osteoporosis-related genes in each KEGG pathway were counted if the Bonferroni-adjusted $p$-value was

4 calculated to be less than 0.05 . $\mathrm{n}$ : the number of pathway-related miRNA target genes. The short bar indicates

5 that there is no miRNA to target any gene involved in the corresponding KEGG pathway. a indicates

6 significantly enriched regulation of pathway-related genes (Bonferroni-adjusted $p<0.05$ ). 